\title{
The influence of the commercial strategy used by aquafeed manufacturers facing the price increase in fish oil on the fatty acid composition of aquafeeds
}

\author{
X. Rollin and Y. Larondelle \\ Unité de Biochimie de la Nutrition, Université de Louvain, Louvain-la-Neuve, Belgium
}

Aquafeeds, the traditional sources of EPA (20:5n-3) and DHA (22:6n-3), contain fish oils obtained from fisheries. Fish oil inclusion in salmonid feeds is currently as high as $25-30 \%(\mathrm{w} / \mathrm{w})$. Given the price fluctuations and the prospect of shortages in the supply of fish oils, some substitution of fish oil with plant oils seems inevitable, but has rarely been studied under practical conditions (commercial feeds).

The present study has analysed in duplicate the proximate and fatty acid (FA) composition of twenty-nine samples (500g each) of salmonid feeds purchased from the two main fish feed manufacturers supplying the Belgium market. The samples were collected between July 2001 and May 2003.

The results showed contrasting FA composition and changes in composition between the two manufacturers. For the first manufacturer (eleven samples) the FA composition was fairly constant throughout the experimental period, with very high EPA + DHA contents (28.3 (SD 4.2) \% total FA) and a rather low linoleic acid (18:2n-6; LA) content (5.1 (SD 1.7) \% total FA). Also, the $n-3: n-6$ was high (5.5 (SD $1.7)$ ) in these feeds. However, as a result of a doubling of the price of fish oil during the experimental period this manufacturer increased the price of feeds by about $€ 51 / \mathrm{t}$ from spring 2002. On the other hand, during the same period the FA composition of the feeds from the second manufacturer (eighteen samples) dramatically changed, with EPA + DHA levels decreasing from 28.4 (SD 2.4 ) \% total FA in late 2001 to 16 (SD 5.1) \% total FA $(P<0.05)$ in early 2003 and LA levels increasing from 6.2 (SD 3.3) \% total FA to 25.4 (SD 7.7 ) \% total FA $(P<0.05)$. The $n-3: n-6$ decreased from 4.9 (SD 1.8) to $<1\left(0.9\right.$ (SD 0.5)). For this manufacturer the determination coefficient $\left(r^{2}\right)$ between the FA composition of the feed and the FA composition of soyabean oil increased from about 0 in late 2001 to 0.71 (SD 0.21 ) in spring 2003 (with extreme values as high as 0.91), suggesting that fish oil in these feeds had been progressively substituted with soyabean oil when the price of fish oil increased. Indeed, during the experimental period this manufacturer did not change the price of the salmonid feeds.

In conclusion, since the FA composition of fish tissues is largely determined by the FA composition of the dietary lipid ingested by the fish, the results raise the question of the control of the FA composition of aquafeeds and its impact on the FA composition of fish from aquaculture available to European consumers. 